\title{
Clinical Features for Severely and Critically III Patients with COVID-19 in Shandong: A Retrospective Cohort Study
}

This article was published in the following Dove Press journal:

Therapeutics and Clinical Risk Management

Shengyu Zhou, ${ }^{1,2, *}$ jiawei Xu, (DD ${ }^{1, *}$ Wenqing Sun, ${ }^{3}$ Jintao Zhang, ${ }^{1}$ Fayan Zhang, ${ }^{4}$ Xuesong Zhao, ${ }^{5}$ Ximing Wang, ${ }^{6}$ Wei Zhang, (iD ${ }^{7}$ Yu Li, Kang Ning, ${ }^{8}$ Yun Pan,' Tian Liu,' Jiping Zhao,' Jiguang Yu, ${ }^{9}$ Yunbo Sun, ${ }^{10}$ Feng Gao, ${ }^{11}$ Rumin Zhang, ${ }^{12}$ Chunsheng Fu, ${ }^{13}$ Yu Sun, ${ }^{14}$ Xiuhe

Ouyang, ${ }^{15}$ Fusen Zhang, ${ }^{16}$ Qing Hu, ${ }^{17}$ Haifeng Teng, ${ }^{18}$ Yun Li, ${ }^{19}$ Chunke Zhang, ${ }^{20}$ Wei Tan, ${ }^{21}$ Jinlai Li, ${ }^{22}$ Lixia Yin, ${ }^{23}$ Liang Dong,' Chunting Wang ${ }^{5}$

'Department of Respiratory and Critical Care Medicine, Qilu Hospital, Cheeloo College of Medicine, Shandong University, Jinan, People's Republic of China; ${ }^{2}$ Clinical Nursing Department, School of Nursing, Cheeloo College of Medicine, Shandong University, Jinan, People's Republic of China; ${ }^{3}$ Intensive Care Unit, Shandong Provincial Chest Hospital, Jinan, People's Republic of China; ${ }^{4}$ College of Traditional Chinese Medicine, Shandong University of Traditional Chinese Medicine, Jinan, People's Republic of China;

Medicine, Jinan, People's Republic of China;
${ }^{5}$ Department of Critical Care Medicine, Shandong

Provincial Hospital Affiliated to Shandong First Medica

University, Jinan, People's Republic of China;

${ }^{6}$ Department of Radiology, Shandong Provincial

Hospital Affiliated to Shandong First Medical

University, Shandong University Jinan, People's

Republic of China; ${ }^{7}$ Department of Lung Disease,

Affiliated Hospital of Shandong University of Traditional

Chinese Medicine, Jinan, People's Republic of China;

${ }^{8}$ The First Affiliated Hospital of Shandong First Medical

University Shandong Provincial Qianfoshan Hospital,

Jinan, People's Republic of China; ${ }^{9}$ Department of

Infectious Diseases, Qishan Hospital, Yantai, People's

Republic of China; ${ }^{10}$ Affiliated Hospital of Qingdao

University, Qingdao, People's Republic of China;

"'Department of Infectious Disease, Linyi People's

Hospital, Linyi, People's Republic of China;

${ }^{12}$ Department of Critical Care Medicine, Zibo Central

Hospital, Zibo, People's Republic of China; ${ }^{13}$ Liaocheng

Contagion Hospital, Liaocheng, People's Republic of

China; ${ }^{14}$ Jining Hospital of Infectious Diseases, Jining

People's Republic of China; ${ }^{15}$ Department of

Respiratory and Critical Medicine, Binzhou People's

Hospital, Binzhou, People's Republic of China;

${ }^{16}$ Intensive Care Unit, Taian City Central Hospital,

Taian, People's Republic of China; ${ }^{17}$ Department of

Respiratory Medicine, Heze Municipal Hospital, Heze

People's Republic of China; ${ }^{18}$ Weihai Municipal

Hospital, Weihai, People's Republic of China; ${ }^{19}$ Jinan

Hospital, Weihai, People's Republic of China; Jinan
Central Hospital, Jinan, People's Republic of China;

${ }^{20}$ Weihai Central Hospital, Weihai, People's Republic of

China; ${ }^{21}$ Department of Respiratory Medicine,

Weifang People's Hospital, Weifang, People's Republic

of China; ${ }^{22}$ Intensive Care Unit, Zaozhuang Municipal

Hospital, Zaozhuang, People's Republic of China;

${ }^{23}$ Department of Respiratory Medicine, Dezhou

People's Hospital, Dezhou, People's Republic of China

*These authors contributed equally to this work

Correspondence: Liang Dong; Chunting Wang Email d15506@I26.com; wcteicu@I26.com
Background: Severe acute respiratory syndrome coronavirus 2 (SARS-CoV-2), a novel pathogen, has caused an outbreak of coronavirus disease 2019 (COVID-19) that has spread rapidly around the world. Determining the risk factors for death and the differences in clinical features between severely ill and critically ill patients with SARS-CoV-2 pneumonia has become increasingly important.

Aim: This study was intended to provide insight into the difference between severely ill and critically ill patients with SARS-CoV-2 pneumonia.

Methods: In this retrospective, multicenter cohort study, we enrolled 62 seriously ill patients with SARS-CoV-2 pneumonia who had been diagnosed by March 12, 2020. Clinical data, laboratory indexes, chest images, and treatment strategies collected from routine medical records were compared between severely ill and critically ill patients. Univariate and multivariate logistic regression analyses were also conducted to identify the risk factors associated with the progression of patients with severe COVID-19.

Results: Of the 62 patients with severe or critical illness, including 7 who died, 30 (48\%) patients had underlying diseases, of which the most common was cardiovascular disease (hypertension, 34\%, and coronary heart disease, 5\%). Compared to patients with severe disease, those with critical disease had distinctly higher white blood cell counts, procalcitonin levels, and D-dimer levels, and lower hemoglobin levels and lymphocyte counts. Multivariate regression showed that a lymphocyte count less than $10^{9} / \mathrm{L}$ (odds ratio 20.92 , 95\% CI 1.76-248.18; $\mathrm{p}=0.02)$ at admission increased the risk of developing a critical illness. Conclusion: Based on multivariate regression analysis, a lower lymphocyte count $\left(<10^{9} / \mathrm{L}\right)$ on admission is the most critical independent factor that is closely associated with an increased risk of progression to critical illness. Age, underlying diseases, especially hypertension and coronary heart disease, elevated D-dimer, decreased hemoglobin, and SOFA score, and APACH score also need to be taken into account for predicting disease progression. Blood cell counts and procalcitonin levels for the later secondary bacterial infection have a certain reference values.

Keywords: SARS-CoV-2, COVID-19, clinical features, severely ill patients, critically ill patients

\section{Background}

On February 11, 2020, a novel virus-associated pneumonia that posed a global health threat was termed COVID-19 (coronavirus disease 2019) by the WHO. This novel infectious virus, which is an enveloped RNA virus, has a similar receptorbinding domain structure to that of severe acute respiratory syndrome coronavirus 
(SARS-CoV), as shown by homology modeling. ${ }^{1}$ As of July 14 , over 570,000 people had died of COVID-19, and the number of confirmed cases had surged to 13.11 million worldwide. In mainland China, more than eighty thousand people were infected with SARS-CoV-2, including 793 in Shandong Province. At present, the focus of this outbreak response is on the treatment of severely ill patients who easily develop critical illness or even die. In China, several studies have described the clinical characteristics and epidemiology of COVID-19 and found that SARS-CoV-2 infection could result in severe and even fatal acute respiratory distress syndrome (ARDS). ${ }^{2-4}$ It has also been reported that the mortality rate of severe cases of COVID-19 is more than $17 \% .^{5}$ Identifying the clinical characteristics of severely ill patients and finding optimal treatment strategies to prevent disease progression is of great significance for reducing mortality and the social impacts of the disease.

Existing articles have shown that some clinical findings, such as high lactate, lymphopenia, and high D-dimer, may suggest a poor prognosis. ${ }^{6-8}$ Some research has suggested that advanced age ( $\geq 75$ years), multiple pulmonary lobe involvement and pleural effusion are associated with a higher disease severity. ${ }^{9}$ This study aimed to identify the most important predictors of disease progression and to provide help for clinicians to improve the effectiveness of the rescue. Additionally, some controversial treatment methods, including gamma globulin, antibiotics, thymosin and glucocorticoids, ${ }^{10,11}$ for severe pneumonia are also discussed in this study.

\section{Methods}

\section{Study Design and Participants}

This retrospective cohort study enrolled 62 seriously ill inpatients (35 males, 27 females) who were almost all from hospitals designated to treat patients with SARSCoV-2 pneumonia in Shandong Province, including Shandong Province Chest Hospital (Jinan, China). These patients who were admitted to hospitals with confirmed SARS-CoV-2 infection were diagnosed with severe or critical disease between January 20 and March 12, 2020. Severity of the disease was classified based on the "Guideline on the management of COVID-19" published by the National Health Commission of the People's Republic of China (version 7.0). Severely ill patients were defined as those who met one of the following conditions: dyspnea with a respiratory rate $\geq 30$ breaths per min, oxygen saturation $\leq 93 \%$, or arterial blood oxygen partial pressure $(\mathrm{PaO} 2)$ /oxygen concentration $(\mathrm{FiO} 2) \leq 40$ $\mathrm{kPa}$. Critically ill patients had one of the following characteristics: respiratory failure requiring mechanical ventilation, shock, or other organ failure requiring intensive care therapy. Sequential Organ Failure Assessment (SOFA) and Acute Physiology and Chronic Health Evaluation (APACHE II) scores are two frequently used scoring systems in intensive care units.

Differences in clinical features, laboratory examinations, CT images and therapies between severe and critical cases were compared. SOFA and APACHE II scores were also compared.

\section{Data Collection}

We reviewed records including routine medical records, nursing records, laboratory, radiological, and microbiological examination results in the electronic medical system for all patients with laboratory-confirmed SARS-CoV-2 infection in Shandong Province and ultimately enrolled 62 severely ill patients.

The data and information collected from patients included sex, age, disease onset date, admission time, duration from admission to severe classification, preexisting conditions, exposure history (exposure to confirmed positive individuals, exposure to suspected positive individuals, or exposure to people from an epidemic area), major defining characteristics for classification as a serious case, clinical manifestations, comorbidities, laboratory examination results (white blood cell count, lymphocyte count, platelet count, creatine kinase, total bilirubin, indirect bilirubin, c-reactive protein, erythrocyte sedimentation rate, procalcitonin, D-dimer, hemoglobin, lactate dehydrogenase, albumin, blood lactate, serum creatinine, and urea nitrogen), imaging features and therapeutic methods (use of ventilation methods, gamma globulin, antibacterial agents, albumin from convalescent plasma, corticosteroids, traditional Chinese medicine, and thymosin).

\section{Specimen Collection and Testing}

Samples for the SARS-CoV-2 test were obtained from oropharyngeal swabs, nasopharyngeal swabs and sputum in all selected patients in accordance with "technical guidelines for COVID-19 laboratory testing, version 2". Pathogen examination was performed using an ORF1ab/N Gene Nucleic acid detection kit (BioGerm, China) following the manufacturer's instructions. The assay targeted the SARS-CoV-2 N gene and ORF1ab gene. A total of $12 \mu \mathrm{L}$ 
nucleic acid amplification reactant, $4 \mu \mathrm{L}$ enzyme mixture, $4 \mu \mathrm{L}$ ORF $1 \mathrm{ab} / \mathrm{N}$ reactant (including primer and probe) and $5 \mu \mathrm{L}$ RNA extracted from the sample were mixed in a suitable centrifuge tube to make the final total fluid volume of the tube reach $25 \mu \mathrm{L}$. Reactions for the amplifications and detection were incubated at $50^{\circ} \mathrm{C}$ for $10 \mathrm{~min}$ and $95^{\circ} \mathrm{C}$ for $5 \mathrm{~min}$, followed by 40 cycles at $95^{\circ} \mathrm{C}$ for $10 \mathrm{~s}$ and $55^{\circ} \mathrm{C}$ for $40 \mathrm{~s}$. After that, melting curve analyses were performed to help exclude nonspecific application. The results interpretation was based on the kit instructions: A cycle threshold value less than or equal to 38 was regarded as a positive test result; otherwise, it was considered a negative test result.

\section{Statistical Analysis}

Statistical analyses were conducted using SPSS version 23.0 (IBM Corporation, New York, NY, USA). Continuous results are expressed as medians and interquartile ranges (IQR), and dichotomous variables are presented as $\mathrm{n}$ (percentage). The Mann-Whitney $U$-test, $\chi^{2}$ test or Fisher's exact test, was performed to calculate the parameter differences, as appropriate. Based on previous articles and existing data, we used age, underlying diseases, lymphocyte count less than $10^{9} / \mathrm{L}$, APACHE II score and SOFA score to perform univariate and multivariate logistic regression analysis. A two-sided $\alpha$ of less than 0.05 was considered statistically significant.

\section{Results}

Data regarding the 62 severely ill patients analyzed in this article were collected from nearly all designated hospitals for COVID-19 treatment in Shandong Province, China (Shandong Provincial Chest Hospital, Yantai Qishan Hospital, etc.). The patients had a median age of 56.5 years (IQR 45.3-64.8) ranging from 25 years to 88 years. Critically ill patients had an older median age (63.0 (51.8-74.8) years) than severely ill patients $(50.5$ (35.8-64.8) years) (Table 1). Thirty-five (56\%) patients were male, and only a few were current smokers or drinkers. Twenty-four (39\%) patients had been exposed to someone from the epidemic area, 18 patients $(36 \%)$ were residents of the area surrounding Wuhan, and 30 (49\%) patients were "close contacts" of positive or suspected patients (suspected patients have fever or respiratory symptoms). A total of 45 cases with confirmed exposure history accounted for $73 \%$ of all cases and of critically ill cases (Table 1). Thirty of 62 severely ill patients were classified as critically ill during hospitalization according to the Chinese management guideline for COVID-19 (version 7.0).

The most common symptoms for all patients were fever $(97 \%)$ and cough $(71 \%)$, followed by sputum production (40\%), dyspnea (35\%), fatigue (24\%) and myalgia $(15 \%)$. The proportions of other symptoms, including diarrhea, nasal congestion or rhinorrhea and pharyngalgia, were all less than or equal to ten percent (Table 1). In critical cases, dyspnea (53\%) and heart rate exceeding 120 per minute $(30 \%)$ occurred more often. Notably, fever was not the first clinical symptom in 15 (24\%) patients whose initial symptoms included cough, expectoration and chest tightness, which are often overlooked. For these nonfebrile patients, the median duration from the onset of symptoms to fever was 3 days (Figure 1). Nearly half of the patients, especially critically ill subjects $(63 \%)$, had comorbidities. Of the eleven comorbidities we investigated, cardiovascular disease $(35 \%)$ was the most common, followed by diabetes (16\%). The frequency of cardiovascular diseases, specifically hypertension $(47 \%$ vs $21 \%$ ) and coronary heart disease $(10 \%$ vs $0 \%)$, was higher in critically ill patients than in severely ill patients. There was no tuberculosis, chronic liver disease or interstitial lung disease found in our cases. Other diseases, such as obesity (3\%), nephropathy (3\%), chronic obstructive pulmonary disease $(3 \%)$, rheumatic immune disease $(2 \%)$ and malignancy (1\%), were also rare (Table 1$)$. The median duration from the onset of symptoms to admission was 6.0 (3.0-9.0) days for all patients, while that from onset of symptoms to severe classification was 9.0 (7.0-13.0) days. For critically ill patients, the time from severe to critical illness was 2.0 (1.0-3.0) days.

Laboratory indexes of blood cell counts, inflammation levels and bodily functions were statistically analyzed. Some indicators, including white blood cell count, hemoglobin, lymphocyte count, D-dimer and procalcitonin, were distinctly different between critically ill and severely ill subjects (Table 2). The higher white cell counts and procalcitonin levels in critically ill patients may to some extent indicate bacterial infection in the later stage of disease. Four indexes (white blood cell count, hemoglobin level, platelet count, and lymphocyte count) extracted from the admission examination before hospitalization were compared to those from the examination at the time when the patients became severely ill (severe or critical cases). For critically ill patients, similar to severely ill patients, lymphocyte counts and hemoglobin levels were significantly lower under severe conditions than at 
Table I Differences in Demographic and Baseline Characteristics Between Patients with Severe and Critical SARS-CoV-2 Pneumonia

\begin{tabular}{|c|c|c|c|c|}
\hline & $\begin{array}{l}\text { All Patients } \\
(n=62)\end{array}$ & $\begin{array}{l}\text { Severely III Patients } \\
(n=32)\end{array}$ & $\begin{array}{l}\text { Critically III Patients } \\
(n=30)\end{array}$ & P value \\
\hline \multicolumn{5}{|l|}{ Demographics and baseline characteristics } \\
\hline Sex & & & & 0.13 \\
\hline Male & $35(56 \%)$ & $21(66 \%)$ & $14(47 \%)$ & \\
\hline Female & $27(44 \%)$ & II(34\%) & $16(53 \%)$ & \\
\hline Age, years & $56.0(45.3-64.8)$ & $50.5(35.8-58.5)$ & $63.0(51.8-74.8)$ & 0.001 \\
\hline $20-39$ & $12(19 \%)$ & $10(31 \%)$ & $2(7 \%)$ & \\
\hline $40-59$ & $25(40 \%)$ & I4(44\%) & $11(37 \%)$ & \\
\hline $60-79$ & $19(301 \%)$ & $7(22 \%)$ & $12(40 \%)$ & \\
\hline$\geq 80$ & $6(10 \%)$ & $\mathrm{I}(3 \%)$ & $5(17 \%)$ & \\
\hline Current smoker & $3(5 \%)$ & $2(6 \%)$ & $\mathrm{I}(3 \%)$ & 0.59 \\
\hline Alcoholic & $9(15 \%)$ & $3(9 \%)$ & $6(20 \%)$ & 0.24 \\
\hline Immunosuppressive therapy history & $\mathrm{I}(2 \%)$ & $0(0 \%)$ & $\mathrm{I}(3 \%)$ & 0.30 \\
\hline Exposure history & $45(73 \%)$ & $23(72 \%)$ & $22(73 \%)$ & 0.90 \\
\hline Exposure to confirmed individuals\# & $21(34 \%)$ & $9(28 \%)$ & $13(43 \%)$ & 0.21 \\
\hline Exposure to individuals from epidemic areas & $24(39 \%)$ & $12(38 \%)$ & $12(40 \%)$ & 0.84 \\
\hline Illness onset preceded fever & $15(24 \%)$ & $8(25 \%)$ & $7(23 \%)$ & 0.88 \\
\hline Days from illness onset to admission & $6.0(3.0-9.0)$ & $5.5(3.0-9.3)$ & $6(3.0-7.0)$ & 0.52 \\
\hline Days from fever to admission & $5.0(2.0-8.0)$ & $4.5(2.0-9.0)$ & $6(2.0-7.0)$ & 0.87 \\
\hline $\begin{array}{l}\text { Days from illness onset to severe } \\
\text { classification }\end{array}$ & $9.0(7.0-13.0)$ & $10.0(7.0-15.2)$ & $9.0(7.0-11.0)$ & 0.26 \\
\hline Days from severe to critical illness & & & $2.0(1.0-3.0)$ & \\
\hline \multicolumn{5}{|l|}{ Signs and symptoms } \\
\hline Fever & $60(97 \%)$ & $32(100 \%)$ & $28(93 \%)$ & 0.14 \\
\hline \multicolumn{5}{|l|}{ Highest temperature, ${ }^{\circ} \mathrm{C}$} \\
\hline$<37.3$ & $2(3 \%)$ & $0(0 \%)$ & $2(7 \%)$ & \\
\hline $37.3-38.0$ & $18(29 \%)$ & $9(28 \%)$ & $9(30 \%)$ & \\
\hline $38.1-39.0$ & $30(48 \%)$ & I4(44\%) & $16(53 \%)$ & \\
\hline$>39$ & $12(19 \%)$ & $9(28 \%)$ & $3(10 \%)$ & \\
\hline Cough & $44(71 \%)$ & $23(72 \%)$ & $21(70 \%)$ & 0.87 \\
\hline Sputum production & $25(40 \%)$ & I4(44\%) & $11(37 \%)$ & 0.54 \\
\hline Myalgia & $9(15 \%)$ & $6(19 \%)$ & $3(10 \%)$ & 0.33 \\
\hline Fatigue & $15(24 \%)$ & $6(19 \%)$ & $9(30 \%)$ & 0.30 \\
\hline Dyspnea (mild or severe) & $22(35 \%)$ & $6(19 \%)$ & $16(53 \%)$ & 0.004 \\
\hline Diarrhea & $6(10 \%)$ & $5(16 \%)$ & $\mathrm{I}(3 \%)$ & 0.10 \\
\hline Nasal congestion or rhinorrhea & $3(5 \%)$ & $2(6 \%)$ & $\mathrm{I}(3 \%)$ & 0.59 \\
\hline Pharyngalgia & $6(10 \%)$ & $3(9 \%)$ & $3(10 \%)$ & 0.93 \\
\hline Systolic pressure $<90 \mathrm{mmHg}$ & $6(10 \%)$ & $\mathrm{I}(3 \%)$ & $5(17 \%)$ & 0.09 \\
\hline Heart rate $>120$ beats per min & $10(16 \%)$ & $\mathrm{I}(3 \%)$ & $9(30 \%)$ & 0.04 \\
\hline Comorbidities & $30(48 \%)$ & II(34\%) & $19(63 \%)$ & 0.02 \\
\hline Diabetes & $10(16 \%)$ & $6(19 \%)$ & $4(13 \%)$ & 0.56 \\
\hline Cardiovascular disease & $22(35 \%)$ & $7(21 \%)$ & $15(50 \%)$ & 0.02 \\
\hline Coronary heart disease & $3(5 \%)$ & $0(0 \%)$ & $3(10 \%)$ & 0.06 \\
\hline Hypertension & $21(34 \%)$ & $7(21 \%)$ & $14(47 \%)$ & 0.04 \\
\hline Chronic obstructive pulmonary disease & $2(3 \%)$ & $\mathrm{I}(3 \%)$ & $\mathrm{I}(3 \%)$ & 0.96 \\
\hline Nephropathy & $2(3 \%)$ & $0(0 \%)$ & $2(7 \%)$ & 0.14 \\
\hline Malignancy & $\mathrm{I}(2 \%)$ & $0(0 \%)$ & $\mathrm{I}(3 \%)$ & 0.30 \\
\hline
\end{tabular}

(Continued) 
Table I (Continued).

\begin{tabular}{|l|l|l|l|l|}
\hline & $\begin{array}{l}\text { All Patients } \\
(\mathbf{n = 6 2 )}\end{array}$ & $\begin{array}{l}\text { Severely III Patients } \\
(\mathbf{n = 3 2 )}\end{array}$ & $\begin{array}{l}\text { Critically III Patients } \\
(\mathbf{n = 3 0})\end{array}$ & P value \\
\hline Rheumatic immune disease & $\mathrm{I}(2 \%)$ & $I(3 \%)$ & $0(0 \%)$ & $2(7 \%)$ \\
Obesity & $2(3 \%)$ & $0(0 \%)$ & $0(0 \%)$ \\
Tuberculosis & $0(0 \%)$ & $0(0 \%)$ & $0(0 \%)$ \\
Chronic liver disease & $0(0 \%)$ & $0(0 \%)$ & $0(0 \%)$ \\
Interstitial lung disease & $0(0 \%)$ & $0(0 \%)$ & 0.14 \\
\hline
\end{tabular}

Notes: Data are presented as the median (IQR), $n(\%)$, or $n / N(\%)$, where $N$ is the total number of patients with available data. $P$ values comparing severely ill patients and critically ill patients are from chi-square test, Mann-Whitney $U$-test, or Fisher's exact test, as appropriate. \#Patients with confirmed SARS-CoV-2 infection.

admission, while the white blood cell counts were higher (Table 3). Lymphocytopenia was a distinct manifestation for COVID-19 patients with poorer health and occurred not only in severely ill patients but also in critically ill patients from admission to when they developed severe disease (severe or critical).

Chest computed tomographic (CT) scans have great value for detecting virus-induced pulmonary injury and viral pneumonia. All patients in our study underwent CT examination during hospitalization. For the imaging features, among 62 patients with COVID-19, 61 (98\%)
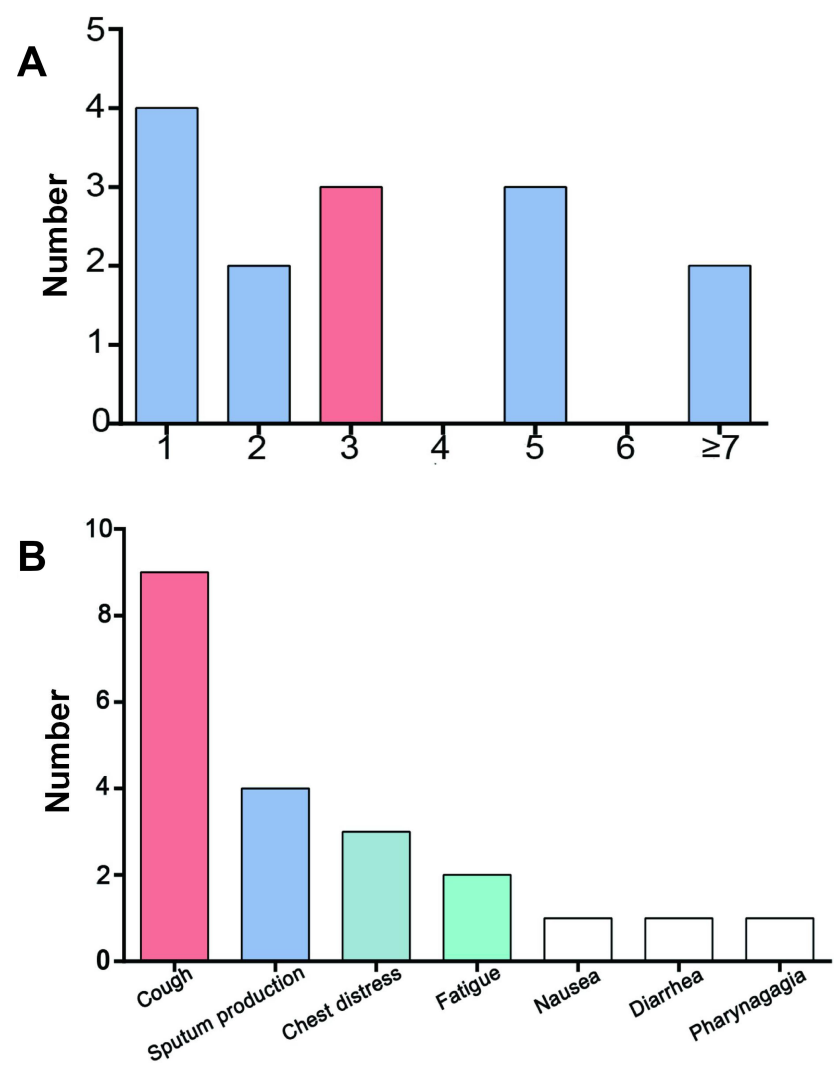

Figure I Characteristics of patients whose initial symptom was not fever. (A) Duration (days) from illness onset to fever for these patients; the median duration was 3 days. (B) Major initial symptoms other than fever. patients had bilateral pulmonary involvement. Patchy opacities $(65 \%)$, ground-glass opacities $(63 \%)$ and consolidation $(47 \%)$ were also noted in many patients' imaging diagnostic reports during the course of the disease (Table 2). We also show the typical CT image evolution of a critically ill patient from admission to disease progression in Figure 2.

The median Acute Physiology and Chronic Health Evaluation II (APACHE II) score and Sequential Organ Failure Assessment (SOFA) score of all cases were 8.0 (IQR 5.0-14.0) and 3.0 (IQR 2.0-5.0), respectively. In our cases, ARDS was the most common outcome, followed by fungal infection, hepatic injury, shock and heart failure, and most of the poor outcomes were frequently observed in critically ill patients (Table 2).

Based on previous findings, we performed logistic regression analysis using several discrepancy indicators (Table 4). Univariate regression analysis showed that older age, underlying disease, higher SOFA score, APACHE II score and fewer lymphocyte counts $\left(<10^{9} / \mathrm{L}\right)$ on admission were more common in severe and critical cases. Multivariate logistic regression analysis showed that a lymphocyte count less than $10^{9} / \mathrm{L}$ on admission was related to the severity of disease (Table 4).

Regarding treatment strategies, 44 (69\%) patients received treatment with nasal cannula, 39 (63\%) with high-flow nasal cannula oxygen therapy, 25 (40\%) with mask ventilation, and $11(18 \%)$ with prone position ventilation (Table 2). Mechanical ventilation and extracorporeal membrane oxygenation can both effectively and rapidly increase the blood oxygen concentration of patients in a poor clinical situation. In total, $15(24 \%)$ and $3(5 \%)$ patients in our study who were critically ill were treated with invasive ventilation or extracorporeal membrane oxygenation, respectively. A total of 56 (90\%) patients received glucocorticoid treatment, and more critically ill 
Table 2 Differences in Clinical Characteristics Between Severely III and Critically III Patients with SARS-CoV-2 Pneumonia

\begin{tabular}{|c|c|c|c|c|}
\hline & All Patients $(n=62)$ & Severely III Patients ( $n=32)$ & Critically III Patients $(n=30)$ & $P$ value \\
\hline \multicolumn{5}{|l|}{ Imaging features } \\
\hline Bilateral pulmonary involvement & $61(98 \%)$ & $31(97 \%)$ & $30(100 \%)$ & 0.33 \\
\hline Unilateral pulmonary involvement & $\mathrm{I}(2 \%)$ & $\mathrm{I}(3 \%)$ & $0(0 \%)$ & 0.33 \\
\hline Pleural effusion & $7(11 \%)$ & $3(9 \%)$ & $4(13 \%)$ & 0.62 \\
\hline Consolidation & $29(47 \%)$ & $14(44 \%)$ & $17(57 \%)$ & 0.31 \\
\hline Patchy opacities & $40(65 \%)$ & $18(56 \%)$ & $23(77 \%)$ & 0.09 \\
\hline Ground-glass opacities & $39(63 \%)$ & $19(59 \%)$ & $20(67 \%)$ & 0.55 \\
\hline Reticulation & $13(21 \%)$ & $6(19 \%)$ & $7(23 \%)$ & 0.66 \\
\hline Honeycombing & $5(8 \%)$ & $2(6 \%)$ & $3(10 \%)$ & 0.59 \\
\hline Crazy paving appearance & $2(3 \%)$ & $\mathrm{I}(3 \%)$ & $\mathrm{I}(3 \%)$ & 0.96 \\
\hline Air bronchograms & $5(8 \%)$ & $2(6 \%)$ & $3(10 \%)$ & 0.59 \\
\hline \multicolumn{5}{|l|}{ Laboratory parameters } \\
\hline White blood cell count, $\times 10^{9}$ per $\mathrm{L}$ & & $5.9(4.6-8.1)$ & $10.8(7.9-15.7)$ & $<0.001$ \\
\hline Hemoglobin, g/L & & $\mid 29.0(|2| .5-\mid 44.0)$ & $117.0(111.5-131.5)$ & 0.006 \\
\hline Platelet count, $\times 10^{9}$ per $\mathrm{L}$ & & $204.0(|7| .0-239.0)$ & $206(163.0-237.0)$ & 0.97 \\
\hline Lymphocyte count, $\times 10^{9}$ per $\mathrm{L}$ & & $0.8(0.6-1.1)$ & $0.6(0.4-0.7)$ & 0.02 \\
\hline Monocyte count, $\times 10^{9}$ per $\mathrm{L}$ & & $0.5(0.2-0.6)$ & $0.5(0.2-0.7)$ & 0.64 \\
\hline C-reactive protein, $\mathrm{mg} / \mathrm{L}$ & & $31.3(\mid 3.5-77.4)$ & $54.1(16.0-86.3)$ & 0.41 \\
\hline Erythrocyte sedimentation rate, $\mathrm{mm} / \mathrm{h}$ & & $59.0(45.0-68.0)$ & $51.0(23.0-63.5)$ & 0.28 \\
\hline D-dimer, $\mu g / L$ & & $0.5(0.4-0.9)$ & $2.6(0.6-8.6)$ & 0.03 \\
\hline Fibrinogen, g/L & & $4.9(3.7-5.9)$ & $4.5(3.6-5.4)$ & 0.18 \\
\hline Procalcitonin, ng/mL & & $0.1(0.0-0.1)$ & $0.2(0.1-0.5)$ & 0.001 \\
\hline Creatine kinase, U/L & & $93.0(60.3-188.3)$ & $92.0(44.5-189.0)$ & 0.70 \\
\hline Creatine kinase isoenzyme, $\mathrm{U} / \mathrm{L}$ & & $15.2(|.2-2| .3)$ & $5.2(1.7-18.0)$ & 0.59 \\
\hline Serum lactate, $\mathrm{mmol} / \mathrm{L}$ & & $2.1(1.2-2.4)$ & $2.4(1.8-3.0)$ & 0.09 \\
\hline Lactate dehydrogenase, U/L & & $303.0(244.5-359.0)$ & $384.8(286.0-454.0)$ & 0.09 \\
\hline Albumin, $g / L$ & & $34.3(31.0-38.0)$ & $35.0(31.3-36.2)$ & 0.85 \\
\hline Total bilirubin, $\mu \mathrm{mol} / \mathrm{L}$ & & $16.9(\mid 2.8-20.4)$ & 19.4(13.7-34.8) & 0.13 \\
\hline Indirect bilirubin, $\mu \mathrm{mol} / \mathrm{L}$ & & $10.8(7.8-13.6)$ & |3.8(5.7-19.3) & 0.49 \\
\hline Serum creatinine, $\mu \mathrm{mol} / \mathrm{L}$ & & $57.8(48.4-67.3)$ & $59.6(40.1-87.7)$ & 0.59 \\
\hline \multicolumn{5}{|l|}{ Treatment strategies } \\
\hline Nasal cannula & $44(69 \%)$ & $22(69 \%)$ & $22(73 \%)$ & 0.69 \\
\hline Mask ventilation & $25(40 \%)$ & $12(38 \%)$ & $13(43 \%)$ & 0.64 \\
\hline High-flow oxygen therapy & $39(63 \%)$ & $13(43 \%)$ & $26(87 \%)$ & $<0.001$ \\
\hline Mechanical ventilation & $25(40 \%)$ & $0(0 \%)$ & $25(83 \%)$ & $<0.001$ \\
\hline Noninvasive mechanical ventilation & $24(45 \%)$ & $0(0 \%)$ & $24(80 \%)$ & $<0.001$ \\
\hline Invasive mechanical ventilation & $15(24 \%)$ & $0(0 \%)$ & $15(50 \%)$ & $<0.001$ \\
\hline Prone-position ventilation & II(I8\%) & $0(0 \%)$ & $11(37 \%)$ & $<0.001$ \\
\hline ECMO & $3(5 \%)$ & $0(0 \%)$ & $3(10 \%)$ & 0.07 \\
\hline Glucocorticoids & $56(90 \%)$ & $26(81 \%)$ & $30(100 \%)$ & 0.01 \\
\hline Albumin & $34(55 \%)$ & $16(50 \%)$ & $15(50 \%)$ & 1.0 \\
\hline Antibiotics & $58(94 \%)$ & $28(88 \%)$ & $30(100 \%)$ & 0.05 \\
\hline Traditional Chinese medicine & $5 I(82 \%)$ & $28(88 \%)$ & $23(77 \%)$ & 0.27 \\
\hline Gamma globulin & $28(45 \%)$ & $14(44 \%)$ & $14(47 \%)$ & 0.82 \\
\hline Convalescent plasma & $4(6 \%)$ & $0(0 \%)$ & $4(13 \%)$ & 0.03 \\
\hline Thymosin & $34(55 \%)$ & $17(53 \%)$ & $17(57 \%)$ & 0.78 \\
\hline \multicolumn{5}{|l|}{ Outcomes } \\
\hline SOFA score & $3.0(2.0-5.0)$ & $2(2.0-3.0)$ & $4(3.0-6.0)$ & 0.003 \\
\hline APACHE II score & $8.0(5.0-14.0)$ & $6.5(4.0-8.3)$ & $12(7.0-19.0)$ & 0.003 \\
\hline
\end{tabular}

(Continued) 
Table 2 (Continued).

\begin{tabular}{|l|l|l|l|l|}
\hline & All Patients (n=62) & Severely III Patients (n=32) & Critically III Patients (n=30) & P value \\
\hline Heart failure & $7(11 \%)$ & $0(0 \%)$ & $7(23 \%)$ & 0.004 \\
Arrhythmia & $6(10 \%)$ & $1(3 \%)$ & $5(17 \%)$ & 0.07 \\
ARDS & $21(34 \%)$ & $2(6 \%)$ & $19(63 \%)$ & $<0.001$ \\
Shock & $7(11 \%)$ & $0(0 \%)$ & $7(23 \%)$ & 0.004 \\
Hepatic injury & $8(13 \%)$ & $2(6 \%)$ & $6(20 \%)$ & 0.11 \\
Fungal infection & $10(16 \%)$ & $2(6 \%)$ & $8(27 \%)$ & 0.03 \\
Multiple organ failure & $5(8 \%)$ & $0(0 \%)$ & $5(17 \%)$ & 0.02 \\
\hline
\end{tabular}

Notes: Data are shown as the median (IQR), $n(\%)$, or $n / N(\%)$, where $N$ is the total number of patients with available data. $P$ values comparing severely ill patients and critically ill patients are from chi-square test, Mann-Whitney U-test, or Fisher's exact test, as appropriate. Laboratory parameters of severely ill patients are collected when their disease state turns to severe, and that of critically ill patients are collected when they have their lowest oxygenation index (data from 7 patients who died were collected when their diseases turn to critical).

patients received glucocorticoids than severely ill patients. In the same period of time, doses of glucocorticoids between the two groups were similar, and no obvious therapeutic effect was observed (Figure 3D-E). Fiftyeight $(70 \%)$ patients were treated with at least one kind of antibiotic, and $51(82 \%)$ were treated with traditional Chinese medicine. Of the 30 critically ill patients, 4 were treated with convalescent plasma, and all survived (Table 2 ). Both gamma globulin and thymosin were administered to the two groups, and there was no significant difference in dose or usage. The therapeutic effect between the group receiving thymosin or gamma globulin and the comparison group showed no obvious differences in recovery time or disease progression (Figure 3A-C). Among several glucocorticoid types, $40 \mathrm{mg}$ methylprednisolone was chiefly utilized when there were increased inflammatory responses and rapid deterioration of the condition. For antibiotics, moxifloxacin and third-generation cephalosporin were used the most frequently (Figure 3).

\section{Discussion}

In this study, critically ill patients were older and had more cardiovascular diseases, including hypertension and coronary heart diseases, lower lymphocyte counts, and higher SOFA scores and APACHE II scores than severely ill patients. Lymphopenia was the only factor associated with fatality for critically ill patients in our research.

Through univariate and multivariate logistic regression, we found that a lymphocyte count less than $10^{9} / \mathrm{L}$ at admission increased the risk of developing severe or critical illness. Strikingly, after comparing patients with different degrees of severity and in different stages of the disease, we found that lymphocyte count was also distinct both in horizontal (severe vs critical) and longitudinal (on admission vs in severe condition) comparisons. This indicated that lymphocytes remained at the normal level in the early stage of SARS-CoV-2 infection, while they decreased to different degrees with progression of the disease. In previous reports, lymphopenia is common in patients with MERS or SARS as a result of innate immune evasion and destruction of lymphocytes by viruses. ${ }^{12-14}$ There are some other studies which had similar result to us. A single-center retrospective study in Wuhan showed that more than $80 \%$ of critically ill patients with COVID19 had lymphopenia. ${ }^{15}$ The proportion of blood lymphocyte has demonstrated the most significant and reliable correlation with disease progression in patients who died due to COVID-19. It also be considered as a valid and accurate indicator for severity of COVID-19 patients, as well as treatment efficacy of COVID-19. ${ }^{16}$ Existed studies proved that lymphopenia accompanied by a decrease in the amount of Treg cells, could cause excessive inflammatory responses. ${ }^{17}$ What's worse, since the specific $\mathrm{T}$ cells acts as an indispensable part in inhibiting coronavirus replication, lymphopenia may support the persistence of the virus. ${ }^{18}$

In COVID-19, the cause of lymphopenia remains unknown, though it has been speculated that perhaps SARS-CoV-2 directly destroys the lymphatic system. ${ }^{16}$ For example, the spleen cell degeneration and necrosis were observed during SARS-CoV-2 infection. ${ }^{19}$ Based on earlier finds regarding SARS and MERS, Some scholars also speculated that SARS-COV-2 can induce apoptosis of lymphocytes. ${ }^{20}$ It was worth mentioning that $\mathrm{p} 53$, as a key pro-apoptosis gene, was highly expressed in PBMCs collected from COVID-19 patients. ${ }^{21}$ In addition, due to the expression of ACE2 on the membrane of hematopoietic stem cells (HSCs), it is considered that SARS-COV-2 can 


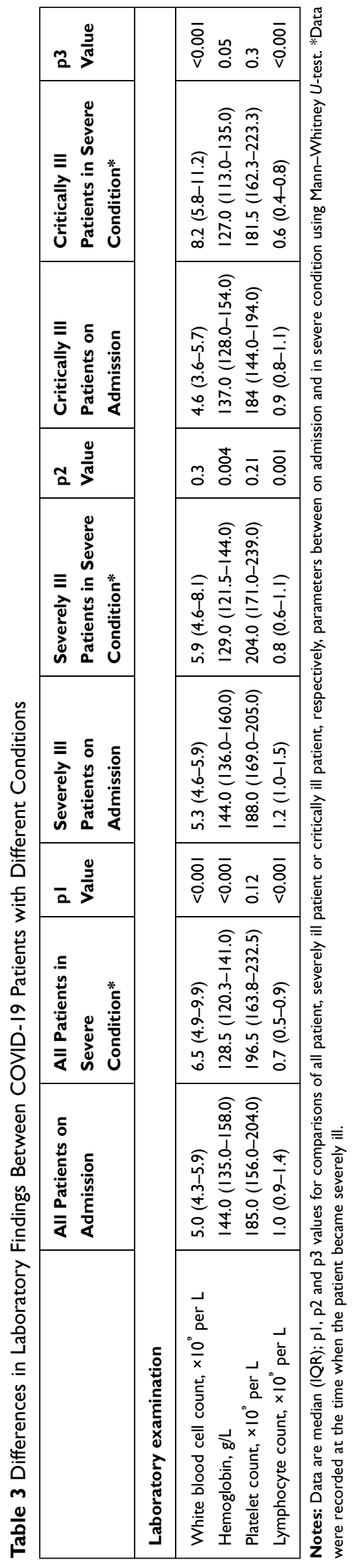

directly infect the pool of HSCs and cause pyroptosis in these cells. ${ }^{22-24}$ The up-regulation of several autophagyassociated genes has also been found in PBMCs collected from COVID-19 patients, suggesting that autophagy mediates the death of lymphocytes. ${ }^{21}$

Therefore, dynamic observation of the lymphocytes count throughout the course of the disease is essential for early identification of the patient's condition. If the lymphocytes progressively decrease to below $10^{9}$, sufficient attention must be paid and necessary measures should be taken early to prevent further deterioration. As a simple and reliable indicator in clinical, lymphocytes count could be used for predicting the severity and outcome of the disease. Further study on the concrete mechanism of lymphopenia and find a way to curb the decrease of lymphocytes may be a promising treatment direction in the future.

Many documents have shown that older age predisposes patients with COVID-19 to critical illness and even death..$^{8,15,25}$ In our data, the age of critically ill patients is generally higher than that of severely ill patients, especially in the 60-79 age group. All the patients who died were older than 55 years old, and the median age was 82 . Critically ill patients are more common among the elderly, which may be related to the weakening of organ function, decreased immunity and more complications. Patients with older age tended to have more complications, such as cardiovascular diseases, and on the basis of these complications, COVID-19 can further increase the risk of visceral insufficiency. ${ }^{26}$ The incidence of hypertension is higher in critically ill patients, which may be related to the higher expression of ACE2 which was considered as the receptor of SARS-CoV-2 in addition to the impairment of cardiac function caused by long-term hypertension.

As for laboratory parameters, D-dimer, which has a proven link with mortality in COVID- $19,{ }^{8}$ increases in critically ill patients. A higher level of D-dimer reflects increased coagulation activity, and combined with underlying cardiovascular disease, it easily causes deterioration during disease. In addition to D-dimer, the white blood cell counts and procalcitonin also increased more obviously in critical cases than in severe cases, which suggests that lung infection was possibly a key factor leading to the deterioration of patients with SARS-CoV-2 at the later stage. Besides, as the embodiment of oxygen carrying capacity, hemoglobin also significantly decreased with the severity of disease as mentioned above. Hemoglobin was distinct both in horizontal (severe vs critical) and longitudinal (on 


\section{A}

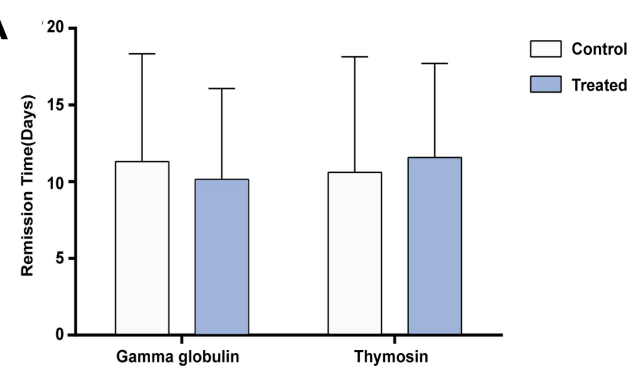

C

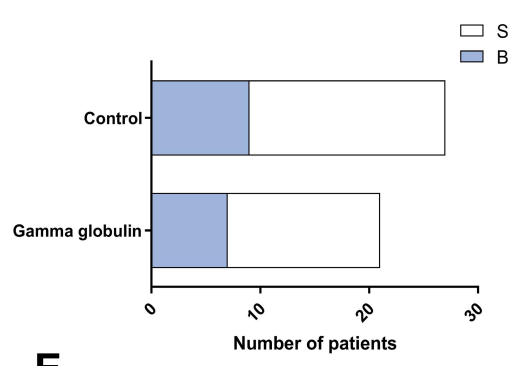

E

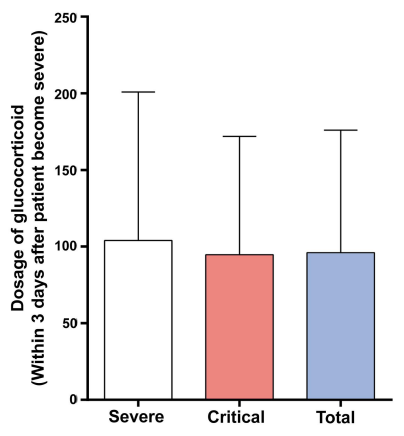

G

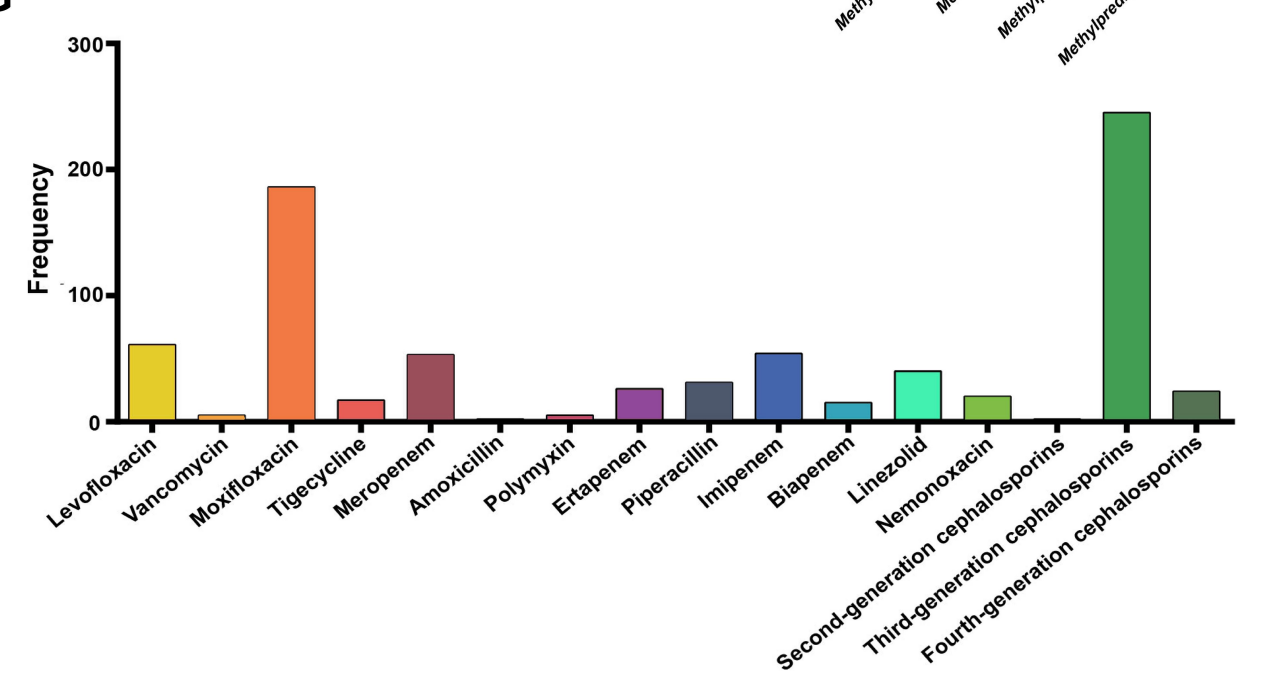

B $\square$ Stay in stable condition

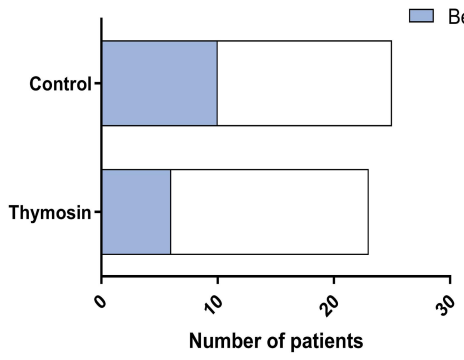

D

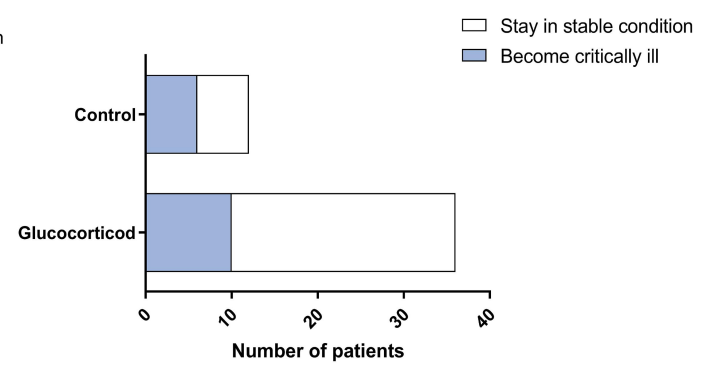

F

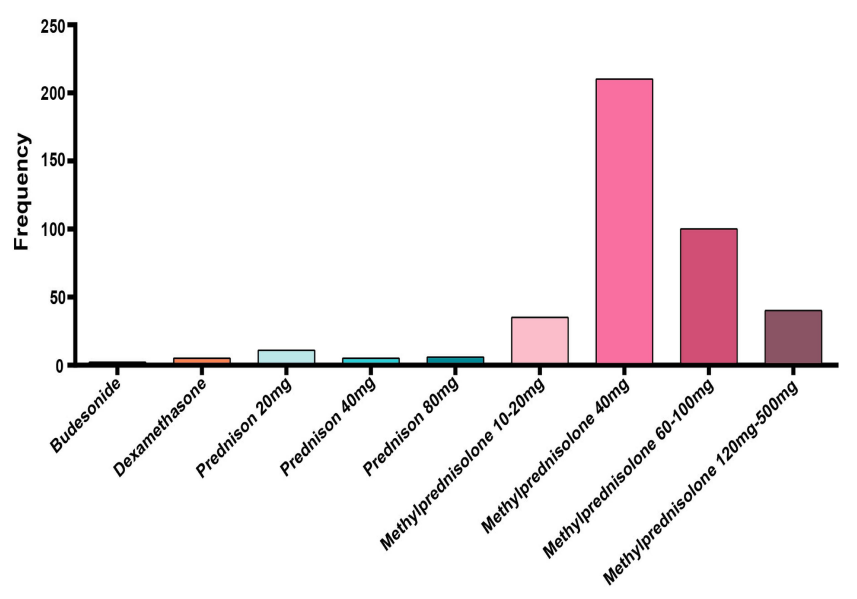

Figure 2 Evolution of chest computed tomographic image findings of a patient with critical COVID-19 during hospitalization. (A) CT image on admission (day 3 after illness onset) showing diffuse bilateral ground glass opacities (GGOs), which were mostly concentrated in the right lung. High-density shadows were distributed in the right inferior lobar bronchus close to the pleura. (B) CT image on the day 9 after illness onset when the patient became severely ill. Large areas of subpleural GGOs and consolidation with air bronchogram signs were observed. (C and D) show the lesions being gradually absorbed on day 19 and 26 after symptom onset. 
Table 4 Regression Analysis of Differential Variables

\begin{tabular}{|c|c|c|c|c|}
\hline & Univariate OR $(95 \% \mathrm{Cl})$ & $\mathrm{p}$ value & Multivariate OR $(95 \% \mathrm{Cl})$ & $\mathrm{p}$ value \\
\hline Age, per year & $1.07(1.03-1.11)$ & 0.002 & $1.06(0.96-1.17)$ & 0.23 \\
\hline Underlying disease (vs no underlying disease) & $3.30(1.16-9.34)$ & 0.03 & $0.62(0.06-6.76)$ & 0.69 \\
\hline \multicolumn{5}{|l|}{ Lymphocyte count, $\times 10^{9}$ per $\mathrm{L}$} \\
\hline$\geq 1.0$ & I (reference) & & & \\
\hline$<1.0$ & $4.76(1.44-15.70)$ & 0.01 & $20.92(1.76-248.17)$ & 0.02 \\
\hline SOFA score & $1.72(1.14-2.60)$ & 0.01 & $1.30(0.85-1.99)$ & 0.22 \\
\hline APACHE II score & $1.20(1.04-1.37)$ & 0.01 & I.I5(0.93-1.44) & 0.21 \\
\hline
\end{tabular}

Abbreviation: OR, odds ratio.

admission vs in severe condition) comparisons. This proved that hemoglobin remained at the normal level in the early stage of SARS-CoV-2 infection, while it decreased to different degrees in combination with disease progression. The decrease in hemoglobin may be related to the destruction of red blood cells, the decrease in food

\section{A}

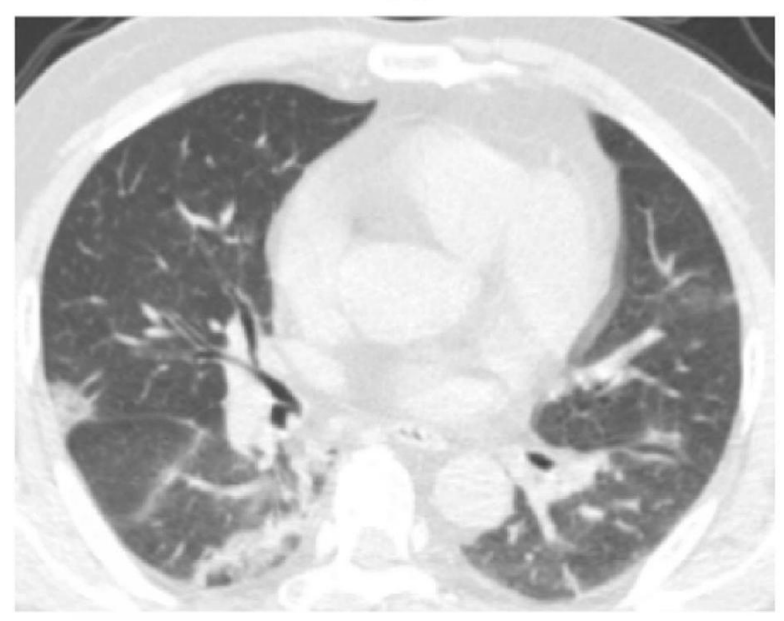

C

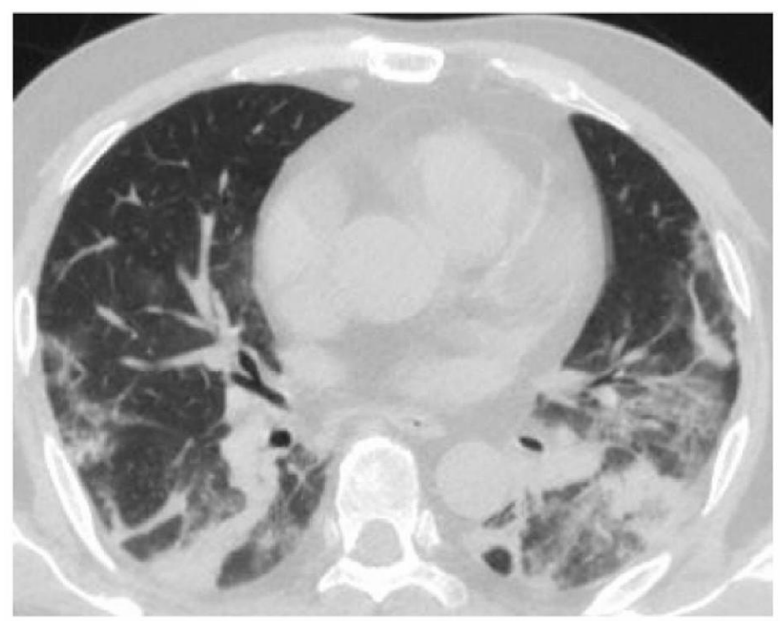

B

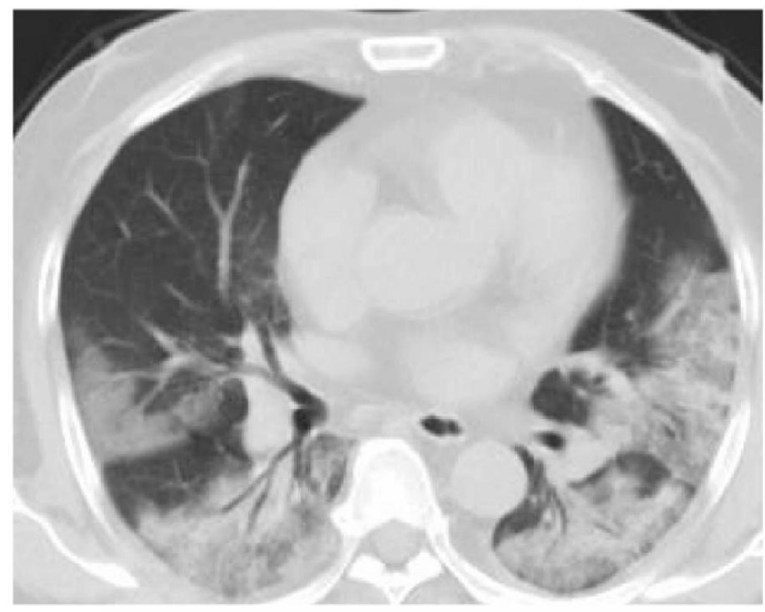

D

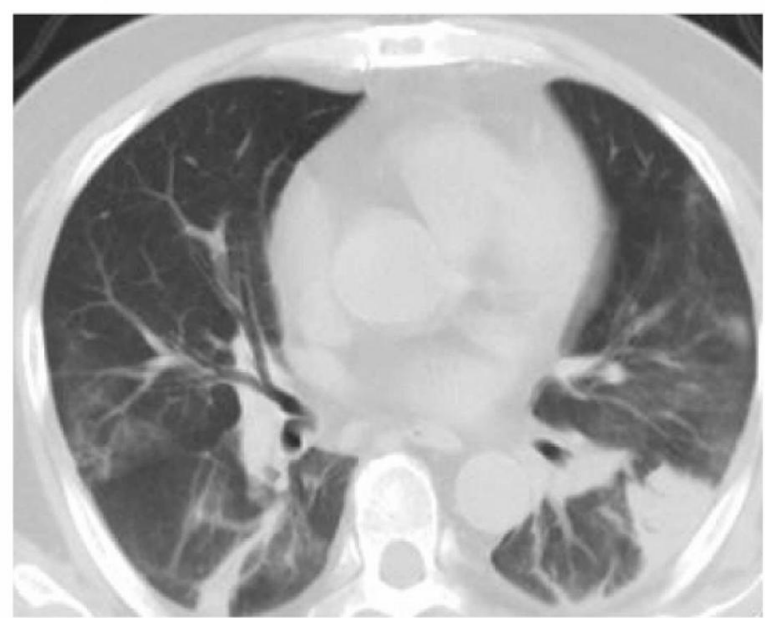

Figure 3 Treatment strategies for patients with severe COVID-19. (A) Recovery time (from severe to moderate disease) between the gamma globulin/thymosin-treated group and the control group. (B-D) Chi-square test comparing control patients and patients treated with thymosin $(p=0.15) / g$ lobulin $(p=1) / g$ lucocorticoid $(p=0.16)$ during the severe illness phase $(14$ patients were excluded because of their short severe stage (less than 2 days)). (E) Dosage of glucocorticoids within 3 days after the diagnosis of severe for the severe and critical groups. (F) Types and frequency of glucocorticoids for treating patients with severe COVID-19. (G) Types and frequency of antibiotics for treating patients with severe COVID-I9. 
intake, poor nutrition or gastrointestinal bleeding in critically ill patients. The reduction of hemoglobin is more pronounced in critically ill patients, leading to less oxygen transportation to the tissues and cells and exacerbation of the damage to the organs. Some studies indicated that a lower level of hemoglobin was associated with a poorer outcome in patients with cardiovascular diseases. $^{27,28}$ The specific reasons need to be further studied.

Higher SOFA and APACHE II scores were frequently observed in critically ill patients. SOFA is a good indicator to evaluate the degree of multiorgan dysfunction. It is useful to predict the occurrence of sepsis, which was often observed in patients with SARS-CoV-2 infection in previous research. ${ }^{8}$ Furthermore, high SOFA levels were considered to be independently associated with mortality in patients hospitalized for COVID-19. ${ }^{29}$ Similar to SOFA, the APACHE II score can also be of importance in the evaluation and prognosis of COVID-19. Both scores were also positively related to the level of lactate dehydrogenase, which is an independent risk factor for severe COVID-19. ${ }^{30}$ Overall, the relationship between the SOFA score, APACHE II score and COVID-19 must be further explained.

Previous studies showed that CT imaging (88\%) had a higher positivity rate than RT-PCR assay (59\%) for the diagnosis of suspected patients with COVID-19 $9^{31}$ and was of great significance in the heavy epidemic area. For severely ill patients with COVID-19, it has also been noted that the CT score can predict the risk of death. ${ }^{32}$ In comparison with other research, ${ }^{3,6}$ patients in our study had more imaging signs and lung lobes involved, which may rely on the severity of disease. Patchy opacities, ground-glass opacities and consolidation were the characteristic imaging findings, which indicated that COVID-19 had invaded and damaged the blood capillaries, resulting in more exudation.

There are several limitations in this study. First, given that differences between "severely ill patients" and "critically ill patients" may be subtle, the number of cases in our study might not be enough to fully reveal all valuable factors and risk factors, such as age and underlying diseases that may be underestimated. Second, because case records in this study come from multiple hospitals, some laboratory parameters inevitably involve missed tests. For this reason, the significance of some parameters, including CRP and lactate, which may play a role in indicating poor prognosis, may be neglected. To ensure the preciseness of the results, we also eliminated some less collected indicators, such as serum ferritin. In the context of a global epidemic, more in-depth studies are urgently needed to clarify the mechanism of COVID-19 and provide possibilities to optimize current supportive means and reduce mortality.

\section{Conclusion}

We confirmed that a lower lymphocyte count $\left(<10^{9} / \mathrm{L}\right)$ on admission is associated with an increased risk of progression to critical illness. Continuous attention to the dynamic changes of lymphocytes is helpful to take early intervention measures to avoid further deterioration and even death. As for other indicators, age, underlying disease (cardiovascular disease), SOFA and APACHE score have certain predictive value for the severity of the disease. Anti-infection therapy should be applied in the late stage of the disease according to WBC count and PCT.

\section{Abbreviations}

COVID-19, coronavirus disease 2019; SARS-CoV-2, severe acute respiratory syndrome coronavirus 2 ; CI, confidence interval; SOFA, Sequential Organ Failure Assessment; APACHE, Acute Physiology And Chronic Health Evaluation; SARS, severe acute respiratory syndrome; ARDS, acute respiratory distress syndrome (ARDS); $\mathrm{PaO} 2$, arterial blood oxygen partial pressure; FiO2, oxygen concentration; CT, computed tomographic; IQR, interquartile range; ACE2, angiotensin converting enzyme II; RT-PCR, reverse transcription-polymerase chain reaction; ECMO, extracorporeal membrane oxygenation.

\section{Data Sharing Statement}

The data used and analyzed during this research are available from the corresponding author (Liang Dong, d15506@126.com) on reasonable request.

\section{Ethics Approval and Consent to Participate}

This study was approved by the ethics committee of Qilu Hospital of Shandong University. This was a retrospective study and all patient data accessed complied with relevant data protection and privacy regulations.

\section{Acknowledgments}

We appreciate Lin Liu (Division of Medical Administration, Health Commission of Shandong 
Province) and Shandong Msunhealth Technology Group Co., Ltd. for providing assistance.

\section{Author Contributions}

LD and CW conceived and designed the study, had full access to all of the data in the study, and take responsibility for the integrity of the data and the accuracy of the data analysis. SZ and JX drafted the paper. SZ, JX, WS, JZ, FZ, and XZ performed the analysis. WS, XW, WZ, YL, KN, YP, TL, JZ, JY, YS, FG, RZ, CF, YS, XO, FZ, QH, HT, YL, CZ, WT, JL and LY collected the data. All authors made substantial contributions to conception and design, acquisition of data, or analysis and interpretation of data; took part in drafting the article or revising it critically for important intellectual content; agreed to submit to the current journal; gave final approval of the version to be published; and agree to be accountable for all aspects of the work.

\section{Funding}

This work was supported by grants from the National Natural Science Foundation of China (81070016, 81270072, 81770029), National Key Research and Development Project (2017YFC1310601) and Key Research Project of Shandong Province (2017GSF218056, 2020SFXGFY03-1, 2020SFXGFY03-3, 2020SFXGFY04). The funders had no role in the study design, data collection, data analysis or preparation of the manuscript.

\section{Disclosure}

The authors declare no conflicts of interest. The authors declare that they have no known competing financial interests or personal relationships that could have appeared to influence the work reported in this paper.

\section{References}

1. Lu R, Zhao X, Li J, et al. Genomic characterisation and epidemiology of 2019 novel coronavirus: implications for virus origins and receptor binding. Lancet. 2020;395(10224):565-574. doi:10.1016/S0140-673 6(20)30251-8

2. Chen N, Zhou M, Dong X, et al. Epidemiological and clinical characteristics of 99 cases of 2019 novel coronavirus pneumonia in Wuhan, China: a descriptive study. Lancet. 2020;395(10223):50 7-513. doi:10.1016/S0140-6736(20)30211-7

3. Wang D, Hu B, Hu C, et al. Clinical characteristics of 138 hospitalized patients with 2019 novel coronavirus-infected pneumonia in Wuhan, China. JAMA. 2020;323(11):1061-1069. doi:10.1001/jama.2020.1585

4. Wu Z, McGoogan JM. Characteristics of and important lessons from the coronavirus disease 2019 (COVID-19) outbreak in china: summary of a report of 72314 cases from the chinese center for disease control and prevention. JAMA. 2020;323(13):1239. doi:10.1001/jama.2020. 2648
5. Grein J, Ohmagari N, Shin D, et al. Compassionate use of remdesivir for patients with severe Covid-19. N Engl J Med. 2020;382 (24):2327-2336. doi:10.1056/NEJMoa2007016

6. Lo IL, Lio CF, Cheong HH, et al. Evaluation of SARS-CoV-2 RNA shedding in clinical specimens and clinical characteristics of 10 patients with COVID-19 in Macau. Int J Biol Sci. 2020;16 (10):1698-1707. doi:10.7150/ijbs. 45357

7. Bhatraju PK, Ghassemieh BJ, Nichols M, et al. Covid-19 in critically ill patients in the seattle region - case series. $N$ Engl J Med. 2020;382(21):2012-2022. doi:10.1056/NEJMoa2004500

8. Zhou F, Yu T, Du R, et al. Clinical course and risk factors for mortality of adult inpatients with COVID-19 in Wuhan, China: a retrospective cohort study. Lancet. 2020;395(10229):1054-1062. doi:10.1016/S0140-6736(20)30566-3

9. Feng Y, Ling Y, Bai T, et al. COVID-19 with different severities: a multicenter study of clinical features. Am J Respir Crit Care Med. 2020;201(11):1380-1388. doi:10.1164/rccm.202002-0445OC

10. Arabi YM, Shalhoub S, Mandourah Y, et al. Ribavirin and interferon therapy for critically ill patients with middle east respiratory syndrome: a multicenter observational study. Clin Infect Dis. 2020;70 (9):1837-1844. doi:10.1093/cid/ciz544

11. Zheng C, Wang J, Guo H, et al. Risk-adapted treatment strategy for COVID-19 patients. Int $J$ Infect Dis. 2020;94:74-77. doi:10.1016/j. ijid.2020.03.047

12. Gu J, Gong E, Zhang B, et al. Multiple organ infection and the pathogenesis of SARS. $J$ Exp Med. 2005;202(3):415-424. doi: $10.1084 /$ jem. 20050828

13. Chu H, Zhou J, Wong BH-Y, et al. Middle east respiratory syndrome coronavirus efficiently infects human primary $\mathrm{T}$ lymphocytes and activates the extrinsic and intrinsic apoptosis pathways. $J$ Infect Dis. 2016;213(6):904-914. doi:10.1093/infdis/jiv380

14. Kindler E, Gil-Cruz C, Spanier J, et al. Early endonuclease-mediated evasion of RNA sensing ensures efficient coronavirus replication. PLoS Pathog. 2017;13(2):e1006195. doi:10.1371/journal.ppat.1006 195

15. Yang X, Yu Y, Xu J, et al. Clinical course and outcomes of critically ill patients with SARS-CoV-2 pneumonia in Wuhan, China: a single-centered, retrospective, observational study. Lancet Respir Med. 2020;8(5):475-481. doi:10.1016/S2213-2600(20)30079-5

16. Tan L, Wang Q, Zhang D, et al. Lymphopenia predicts disease severity of COVID-19: a descriptive and predictive study. Signal Transduct Target Ther. 2020;5(1):33. doi:10.1038/s41392-0200148-4

17. Qin C, Zhou L, Hu Z, et al. Dysregulation of immune response in patients with coronavirus 2019 (COVID-19) in Wuhan, China. Clin Infect Dis. 2020;71(15):762-768. doi:10.1093/cid/ciaa248

18. Zhao J, Zhao J, Perlman S. T cell responses are required for protection from clinical disease and for virus clearance in severe acute respiratory syndrome coronavirus-infected mice. J Virol. 2010;84 (18):9318-9325. doi:10.1128/jvi.01049-10

19. Yao X, Li TY, He ZC, et al. [A pathological report of three COVID-19 cases by minimal invasive autopsies].. Zhonghua Bing Li Xue Za Zhi. 2020;49(5):411-417. doi:10.3760/cma.j.cn11215120200312-00193

20. Jafarzadeh A, Jafarzadeh S, Nozari P, Mokhtari P, Nemati M. Lymphopenia an important immunological abnormality in patients with COVID-19: possible mechanisms. Scand J Immunol. 2020; e12967. doi:10.1111/sji.12967

21. Xiong Y, Liu Y, Cao L, et al. Transcriptomic characteristics of bronchoalveolar lavage fluid and peripheral blood mononuclear cells in COVID-19 patients. Emerg Microbes Infect. 2020;9 (1):761-770. doi:10.1080/22221751.2020.1747363

22. Singh N, Joshi S, Guo L, et al. ACE2/Ang-(1-7)/Mas axis stimulates vascular repair-relevant functions of CD34+ cells. American Journal of Physiology-Heart and Circulatory Physiology. 2015;309(10): H1697-1707. doi:10.1152/ajpheart.00854.2014 
23. Jarajapu Y. Targeting ACE2/angiotensin-(1-7)/mas receptor axis in the vascular progenitor cells for cardiovascular diseases. $\mathrm{Mol}$ Pharmacol. 2020;99(1):29-38. doi:10.1124/mol.119.117580

24. Ratajczak M, Kucia M. SARS-CoV-2 infection and overactivation of Nlrp3 inflammasome as a trigger of cytokine "storm" and risk factor for damage of hematopoietic stem cells. Leukemia. 2020;34 (7):1726-1729. doi:10.1038/s41375-020-0887-9

25. de Wit E, van Doremalen N, Falzarano D, Munster VJ. SARS and MERS: recent insights into emerging coronaviruses. Nat Rev Microbiol. 2016;14(8):523-534. doi:10.1038/nrmicro.2016.81

26. Guan W-J, Ni Z-Y, Hu Y, et al. Clinical characteristics of coronavirus disease 2019 in China. N Engl J Med. 2020;382(18):1708-1720. doi:10.1056/NEJMoa2002032

27. Anker SD, Voors A, Okonko D, et al. Prevalence, incidence, and prognostic value of anaemia in patients after an acute myocardial infarction: data from the OPTIMAAL trial. Eur Heart J. 2009;30 (11):1331-1339. doi:10.1093/eurheartj/ehp116

28. Cavusoglu E, Chopra V, Gupta A, et al. Usefulness of anemia in men as an independent predictor of two-year cardiovascular outcome in patients presenting with acute coronary syndrome. Am J Cardiol. 2006;98(5):580-584. doi:10.1016/j.amjcard.2006.03.031
29. Rivera-Izquierdo M, Del Carmen Valero-Ubierna M, R-delAmo JL, et al. Sociodemographic, clinical and laboratory factors on admission associated with COVID-19 mortality in hospitalized patients: a retrospective observational study. PLoS One. 2020;15(6): e0235107. doi:10.1371/journal.pone.0235107

30. Han Y, Zhang H, Mu S, et al. Lactate dehydrogenase, an independent risk factor of severe COVID-19 patients: a retrospective and observational study. Aging (Albany NY). 2020;12(12):11245-11258. doi:10.18632/aging.103372

31. Ai T, Yang Z, Hou H, et al. Correlation of chest CT and RT-PCR testing for coronavirus disease 2019 (COVID-19) in China: a report of 1014 cases. Radiology. 2020;296(2):E32-E40. doi:10.1148/ radiol.2020200642

32. Yuan M, Yin W, Tao Z, Tan W, Hu Y, Schildgen O. Association of radiologic findings with mortality of patients infected with 2019 novel coronavirus in Wuhan, China. PLoS One. 2020;15(3): e0230548. doi:10.1371/journal.pone.0230548

\section{Publish your work in this journal}

Therapeutics and Clinical Risk Management is an international, peerreviewed journal of clinical therapeutics and risk management, focusing on concise rapid reporting of clinical studies in all therapeutic areas, outcomes, safety, and programs for the effective, safe, and sustained use of medicines. This journal is indexed on PubMed Central, CAS,
EMBase, Scopus and the Elsevier Bibliographic databases. The manuscript management system is completely online and includes a very quick and fair peer-review system, which is all easy to use. Visit http://www.dovepress.com/testimonials.php to read real quotes from published authors. 